\title{
Optimal Voltage Regulation for Unbalanced Distribution Networks Considering Distributed Energy Resources
}

\author{
Guodong Liu*, Oğuzhan Ceylan ${ }^{\dagger}$, Yan $\mathrm{Xu}^{*}$ and Kevin Tomsovic ${ }^{\dagger}$ \\ * Power and Energy Systems Group, Oak Ridge National Laboratory \\ Oak Ridge, Tennessee 37381 \\ Email: liug@ornl.gov, xyu3@ornl.gov \\ ${ }^{\dagger}$ Department of Electrical Engineering and Computer Science \\ University of Tennessee, Knoxville, Tennessee 37996 \\ Email: oceylan@utk.edu, tomsovic@ tennessee.edu
}

\begin{abstract}
With increasing penetration of distributed generation in the distribution networks (DN), the secure and optimal operation of DN has become an important concern. In this paper, an iterative quadratic constrained quadratic programming model to minimize voltage deviations and maximize distributed energy resource (DER) active power output in a three phase unbalanced distribution system is developed. The optimization model is based on the linearized sensitivity coefficients between controlled variables (e.g., node voltages) and control variables (e.g., real and reactive power injections of DERs). To avoid the oscillation of solution when it is close to the optimum, a golden search method is introduced to control the step size. Numerical simulations on modified IEEE 13 nodes test feeders show the efficiency of the proposed model. Compared to the results solved by heuristic search (harmony algorithm), the proposed model converges quickly to the global optimum.

Index Terms-Voltage regulation, sensitivity coefficients, linearization, distributed energy resource (DER), golden search method, unbalanced distribution network.
\end{abstract}

\section{INTRODUCTION}

With increasing penetration of distributed energy resources (e.g., wind turbines, PV panels, microturbines, fuel cells, minihydro, battery storage, and so on) in the distribution networks (DN), the traditional passive DNs without any sources are gradually transforming into active ones with both dispatchable and non-dispatchable power sources[1]. Correspondingly, the usual "install and forget" principle becomes infeasible and

This work was sponsored by the Office of Electricity Delivery \& Energy Reliability, U.S. Department of Energy under Contract No. DE-AC05-00OR 22725 with UT-Battelle and conducted at ORNL and UT Knoxville. This work also made use of Engineering Research Center Shared Facilities supported by the Engineering Research Center Program of the National Science Foundation and the Department of Energy under NSF Award Number EEC-1041877 and the CURENT Industry Partnership Program. The second author would like to thank the Scientific and Technological Research Council of Turkey (TUBITAK) for its financial support.

Guodong Liu and Yan Xu are with the Oak Ridge National Laboratory (ORNL), Oak Ridge, TN 37831 USA (email: liug@ornl.gov; xuy3@ornl.gov).

Oğuzhan Ceylan and Kevin Tomsovic are with the Department of Electrical Engineering and Computer Science, The University of Tennessee (UT), Knoxville, TN 37996 USA (email: oceylan@utk.edu; tomsovic@utk.edu). potentially could compromise operating efficiency. Within this context, new control and operation strategies capable of coordinating different types of distributed energy resources (DERs) efficiently to achieve operational objectives are in particular need of development [2].

Management and optimizing the operation of these active DNs require a full AC-formulation of the power flow equations. Various approaches have been proposed for solving the distribution optimal power flow (DOPF) problem in literature. Generally, these approaches can be classified into two categories. In the first category, the nonlinear optimization problem is directly solved using nonlinear programming methods such as gradient search or interior point methods [3]-[6]. The solution time and convergence characteristic of nonlinear programming in solving the DOPF problem may not satisfy the stringent time constraints required by real-time controls. More commonly, as in the second category, the nonlinear optimization problem is addressed by iteratively solving the linearized problem [7]-[12]. These linear programming based methods are generally more efficient in term of solution time. Nevertheless, the linearized problem is formulated based on the calculated sensitivity coefficients. When the solution is close to the optimum, the linearized sensitivities do not represent the nonlinear system correctly. In this case, the solution may oscillate around the optimum and fail to converge.

Traditionally, voltage profile and power flow in distribution feeders have been locally controlled using switched devices such as shunt capacitor banks and voltage regulators. These devices are expected to switch only a few times a day to accommodate relatively slow variations in load. Thus, they cannot effectively respond to mitigate the frequent voltage deviations caused by the rapid output fluctuations of renewable resources. Under this circumstance, inverter-interfaced DERs can provide dynamic voltage/VAR support to maintain system voltage stability and improve voltage profile [13]-[16]. The well known apparent power equation, $S^{2}=P^{2}+Q^{2}$, shows that a small change in active power output of DERs can 
actually provide a wide range of reactive power capability.

This paper is a direct extension of [15], [16] with improvements in multiple aspects. The main contributions of this paper are as follows.

1) A nonlinear one dimensional optimization problem is added to determine the optimal step size of the control variables. The problem is solved by the golden search method. By this way, the oscillation of solution is avoided and the convergence is accelerated.

2) In sensitivity calculation [17], various types of power injections (i.e. constant PQ, constant impedance and constant current) are taken into account and the accuracy of calculation is improved by removing the assumption of constant PQ injections as in [15], [16].

3) A population based heuristic search method, harmony search, is also used to solve the problem [18]. The results indicate that the proposed linearized model is indeed converging to the global optimum.

The rest of this paper is organized as follows. In Section II, the quadratic constrained quadratic programming (QCQP) formulation for the linearized optimization problem of voltage regulation is presented. The golden search method is integrated to accelerate the convergence. In Section III, the proposed model is validated on modified IEEE 13 nodes test feeders and the results are compared to those of the harmony search based optimization. Section IV summarizes the paper and presents conclusions.

\section{Formulation AND METHODOLOGY}

In this section, the QCQP formulation for the linearized optimization problem of voltage regulation is presented. Then, the golden search method is introduced to avoid the oscillation$\mathrm{s}$ when close to the optimum. After that, the harmony search algorithm is introduced.

\section{A. QCQP Formulation for Optimal Voltage Regulation}

In distribution systems operation, one wants the bus voltage magnitudes as close as possible to $1 \mathrm{pu}$. Large voltage deviations may cause inefficiencies and other problems. Other considerations in the objective function is maximizing active power output of inverter based DER. The optimal voltage regulation problem is formulated as a QCQP. The controlled variables are the node voltages and the control variables are the active and reactive power injections of the DERs. The objective of the optimal voltage regulation problem is:

$$
\min _{\triangle x} \sum_{i=1}^{3 N_{G}}\left(-\triangle P_{i}^{D E R}\right)+\sum_{j=1}^{3 N_{B}}\left(\left|V_{j}\right|^{(k)}+\triangle\left|V_{j}\right|-1\right)^{2}
$$

where $\triangle x=\left[\triangle P^{D E R}, \triangle Q^{D E R}\right]$ is the control variable vector. $N_{G}$ is the number of DERs and $N_{B}$ is the number of nodes. The linearized sensitivities directly link node voltages with the control variables. In this way, the complicated distribution power flow equations are replaced by the simple linear equation as following:

$$
\triangle\left|V_{j}\right|=\sum_{i=1}^{3 N_{G}}\left(K_{i j}^{P} \triangle P_{i}^{D E R}+K_{i j}^{Q} \triangle Q_{i}^{D E R}\right)
$$

where $K_{i j}^{P}$ and $K_{i j}^{Q}$ are the sensitivity coefficients of voltage deviations at node $j$ to control variables variations $\triangle P_{i}^{D E R}$ and $\triangle Q_{i}^{D E R}$. Other constraints are included as following:

$$
\begin{gathered}
0.95 \leq\left|V_{j}\right|^{(k)}+\triangle\left|V_{j}\right| \leq 1.05 \quad \forall j \\
P_{i}^{D E R(k)}+\triangle P_{i}^{D E R} \leq P_{i}^{\max } \forall i \\
\left(P_{i}^{D E R(k)}+\triangle P_{i}^{D E R}\right)^{2}+\left(Q_{i}^{D E R(k)}+\triangle Q_{i}^{D E R}\right)^{2} \leq S_{i}^{2} \forall i
\end{gathered}
$$

where $P_{i}^{\max }$ is the maximum real power output of DER $i$ and $S_{i}$ is the capacity of DER $i$ or the inverter connected.

A QCQP algorithm is used to solve the optimization problem (1-5). Hence, the improvement direction $\triangle x$ of the control variable vector $x=\left[P^{D E R}, Q^{D E R}\right]$, with $P^{D E R}$ and $Q^{D E R}$ being the active and reactive power set-points of all DERs, are determined. The initial values of the DERs' control variables are modified by $\triangle x$ and the new improvement direction is calculated based on the updated sensitivity coefficients at current operating condition. This iterative procedure continues until the objective function or the control variables do not change significantly between two consecutive iterations or a maximum number of iterations is reached.

It should be noted that the quadratic capacity constraint (5) can also be linearized. With linear objective function, e.g., summation of absolute value of voltage deviations, the QCQP can be adapted to linear programming, which has much higher solution efficiency.

\section{B. Golden Search Method for Avoiding Oscillations}

The QCQP problem is formulated based on the calculated sensitivity coefficients. When the operating point is far from the optimum, the solution of the QCQP gives a reasonable improvement direction $\triangle x$ since the sensitivity coefficients represent the nonlinear system within a small error range. However, as the solution approaches the optimum, the nonlinearity of system increases. When the solution is close to the optimum, the sensitivity coefficients can change dramatically. In fact, their signs may change at the optimum. In this case, the linearized sensitivities do not represent the nonlinear system correctly. As a result, the solution oscillates around the optimum and may not converge as shown in Fig. 1.

To avoid the oscillations when close to the optimum, a golden search method is introduced to control the step size of $\triangle x$. After the calculation of the $k+1$ iteration, the elements of improvement direction $\triangle x^{(k+1)}$ are compared with that of the last iteration $\triangle x^{(k)}$. If any pair of them has a different sign, an oscillation is detected. Then, a nonlinear one dimensional optimization problem is solved by the golden search method to determine the optimal step size $\xi$ of the control variables. The improvement direction $\triangle x^{(k+1)}$ is modified by $\xi \triangle x^{(k+1)}$. In this way, the oscillations are avoided and convergence 


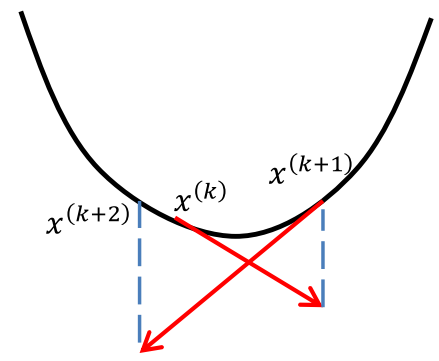

Fig. 1: Oscillation of iterations near the optimum

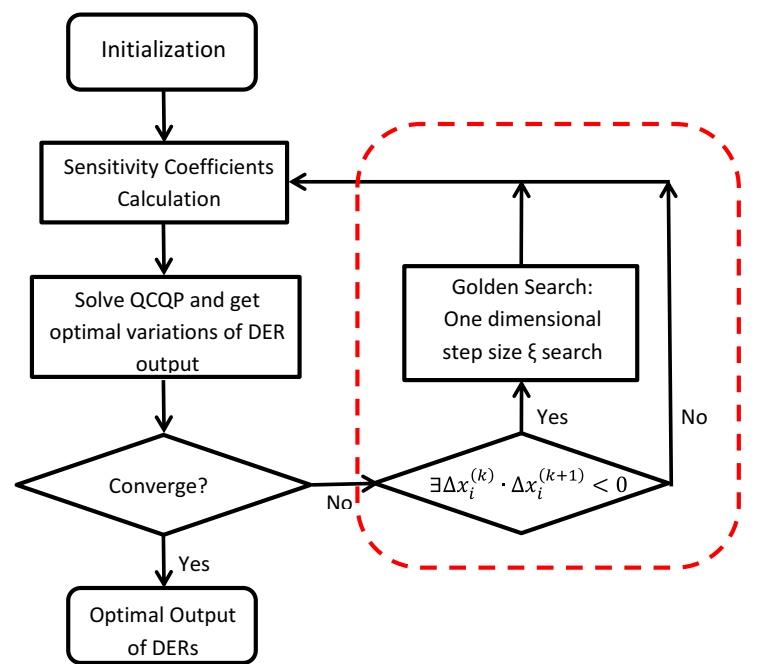

Fig. 2: Iterative optimization procedure with golden search method

accelerates. The iterative optimization procedure with golden search methods shown is Fig. 2

\section{Harmony Search Algorithm}

The harmony search algorithm is inspired by the way jazz musicians seek to find better harmony by adjusting their playing during improvisation. Like other derivative free, population based algorithms, this method initially creates a random solution vector in the feasible solution space and with the help of operators like mutation and crossover, the solutions of the problem improve and the global optimum can be found given an infinite number of iterations. The optimization problem is as follows:

$$
\begin{gathered}
\min _{x} \sum_{i=1}^{3 N_{G}}\left(-P_{i}^{D E R}\right)+\sum_{j=1}^{3 N_{B}}\left(\left|V_{j}\right|-1\right)^{2} \\
\text { s.t. } 0.95 \leq\left|V_{j}\right| \leq 1.05 \quad \forall j \\
P_{i}^{D E R} \leq P_{i}^{\max } \quad \forall i \\
\left(P_{i}^{D E R}\right)^{2}+\left(Q_{i}^{D E R}\right)^{2} \leq S_{i}^{2} \quad \forall i
\end{gathered}
$$

The method consists of four steps: initialization of solution candidate, improvisation, update process and control of stopping criterion.
1) Choose harmony memory size (HMS), harmony memory consideration rate (HMCR) and pitch adjusting rate (PAR). Create initial solution candidates and compute their objective function values.

2) Create a new solution candidate vector by performing the following operations:

- Generate a random number between 0 and 1 , and compare it with harmony memory consideration rate (HMCR). If this number is bigger than HMCR create a random solution candidate vector.

- If this number is smaller than HMCR, construct a new solution vector by randomly picking elements from the candidate solution matrix one by one. At each step of this process construct two random numbers. Compare the first one with pitch adjusting rate (PAR). If it is bigger than PAR do nothing, otherwise either increase or decrease the solution candidate element by a small value.

3) Compute the value of objective function of the new solution candidate vector, then compare the value of the objective function of the new solution candidate vector with the worst objective function of the solution candidate matrix. If it is smaller replace the row of the solution candidate matrix with the new solution candidate vector, otherwise do nothing.

4) Finished if stopping criterion is reached, otherwise continue from step 2.

It should be noted that the efficiency of harmony search algorithm is much lower compared with the proposed iterative optimization, but since it can converge to a global optimum given sufficient time, it is used to check whether the proposed iterative optimization procedure can find the global optimum.

\section{CASE STUdies}

The proposed iterative optimization procedure is demonstrated on a modified IEEE 13 nodes test feeder as shown in Fig. 3 [19]. In the modified system, the real power load at node 671 is tripled for each phase. Three DERs with a capacity of $770 \mathrm{kVA}$ are installed at bus 671 (one for each phase). Thus, the increased load is balanced out if the DERs real power output reaches its full capacity. To create 40 different scenarios, the available active power output of DERs is decreased from maximum to zero output in 5\% decrement and then increased back to maximum. For these scenarios, 3 cases are studied:

- Case 1: Only a voltage regulator is considered. The real power outputs of DERs are assumed to be at maximum for all scenarios, and power factor is assumed to be 1 .

- Case 2: The tap positions of voltage regulator are fixed at its nominal value [ $\left.\begin{array}{lll}9 & 6 & 8\end{array}\right]$, but DERs are allowed to generate/consume reactive power, i.e., power factor is not fixed. The problem is solved by the proposed iterative optimization procedure.

- Case 3: Same as case 2 but solution is found by harmony search algorithm with HMS $=20, \mathrm{HMCR}=0.8$ and PAR $=0.4$. 


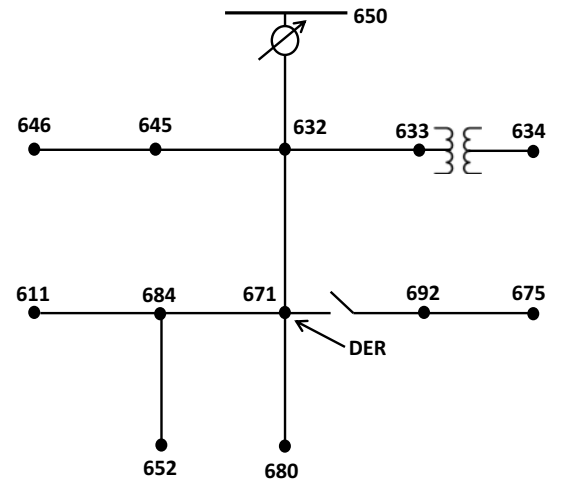

Fig. 3: Modified IEEE 13 nodes test feeder

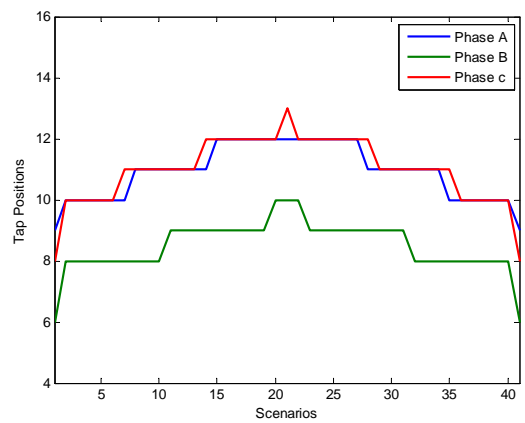

Fig. 4: Tap positions of Case 1

All numerical simulations are coded in MATLAB. The QCQP in Case 2 is solved using the QCP solver in CPLEX 12.2. With a pre-specified maximum error of $0.01 \mathrm{kVA}$, the running time of each scenario is about $4 \mathrm{~s}$ on a $2.66 \mathrm{GHz}$ Windows-based PC with $4 \mathrm{~Gb}$ of RAM.

In Case 1, the tap positions of voltage regulator for different scenarios are shown in 4. As can be seen, the tap positions change as the power output of DERs varies. Since the power outputs of renewable DERs are subject to rapid and frequent fluctuations, and voltage regulators are expected to switch only a few times a day to accommodate relatively slow variations in load, hence the voltage regulator would probably not be able to effectively respond to mitigate the frequent voltage deviations caused by DERs.

In Case 2, the DERs are allowed to generate/consume reactive power. The real and reactive power outputs of DERs are shown in Fig. 5. As the real power output of DERs decreases, the load increases and the voltage drop on the feeders increases. To reduce the voltage drop and support the node voltages, the reactive power output of DERs increases. In particular, the reactive power output of phase B is negative in some scenarios. This is because phase B is close to overvoltage in these scenarios, which can be verified by Fig. 6 .

The voltages of Case 1 and Case 2 are compared in Fig. 6 The vertical bars show the range of voltages and the mark on each bar shows the average value. The nodes are renumbered as 1(650a), 2(632), 3(632a), 4(671), 5(684), 6(652), 7(633), 8(634), 9(645), 10(646), 11(692), 12(675),
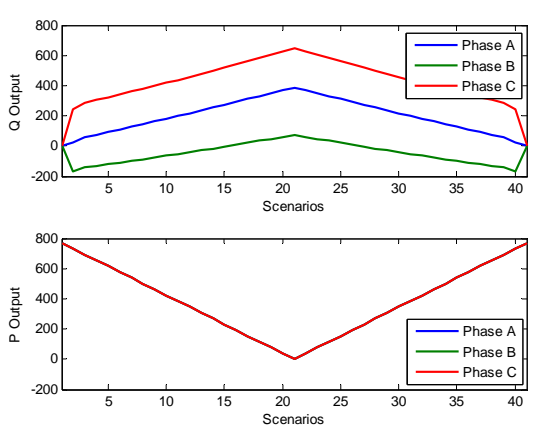

Fig. 5: Real and reactive power output of DERs in Case 2

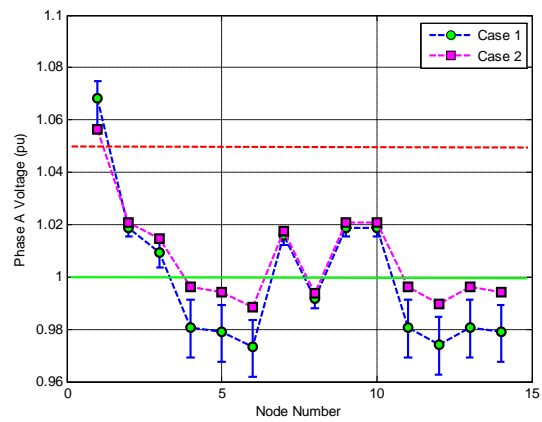

(a) Phase A

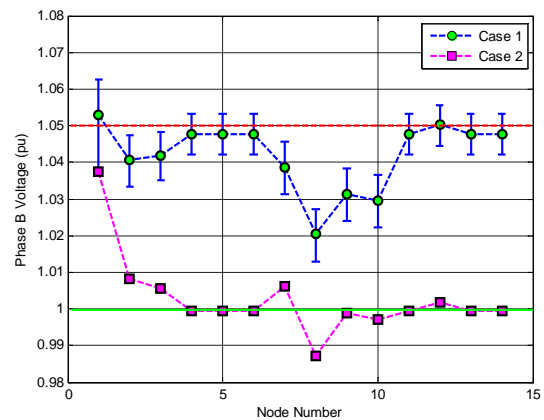

(b) Phase B

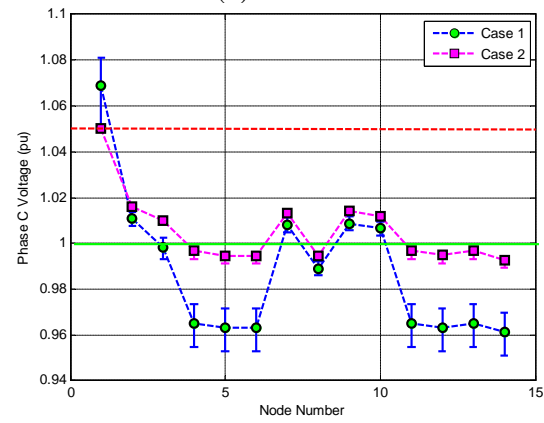

(c) Phase C

Fig. 6: Comparison of voltages in Case 1 and 2

13(680) and 14(611) for simplicity. As can be seen, the node voltages in Case 2 are much closer to 1 than Case 1. Also, the node voltages of Case 2 almost stay constant while the real power outputs of DERs change in different scenarios. Thus, even if the voltage regulator can be switched frequently in Case 1, voltage profiles can still be improved significantly in Case 2 with DERs providing dynamic Var support. 


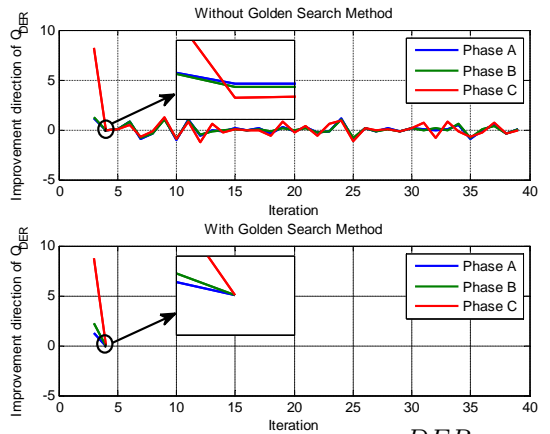

Fig. 7: Change in reactive power $\triangle Q^{D E R}$ across iterations
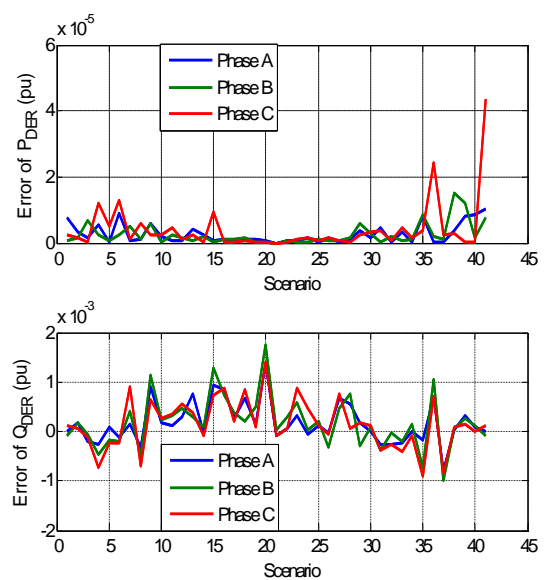

Fig. 8: Comparison of Case 2 and 3 results

In order to show the effect of integrated golden search method, the improved direction of reactive power $\triangle Q^{D E R}$ for the scenario with zero active power output is compared in Fig. 7. As can be seen, without the golden search method, $\triangle Q^{D E R}$ oscillates and does not converge. With the golden search method, $\triangle Q^{D E R}$ is modified by optimal step size $\xi$ and the solution converges after 4 iterations.

In order to check whether the proposed method converges to global optimum, the real and reactive power of DERs calculated in Case 2 and Case 3 are compared. The relative differences (based on the capacity of DERs) are shown in Fig. 8. As can be seen, both the differences of real power and reactive power of DERs are very small. The proposed iterative optimization procedure converges to the global optimum for these cases.

\section{CONCLUSIONS}

In this paper, an iterative QCQP model to minimize voltage deviations and maximize DERs active power output in a three phase unbalanced distribution system is proposed. A nonlinear one dimensional optimization problem solved by golden search method is integrated into an iterative QCQP approach. Numerical simulations on a modified IEEE 13 nodes test feeder show the efficiency of the proposed model. The voltage profiles can be significantly improved with DERs providing dynamic Var support. The proposed model finds the global optimum for the test cases. Case studies on larger systems with multiple DERs will be studied in the future work.

\section{REFERENCES}

[1] J. A. P. Lopes, N. Hatziargyriou, J. Mutale, P. Djapic, and N. Jenkins, "Integrating distributed generation into electric power systems: A review of drivers, challenges and opportunities," Elect. Power Syst. Res., vol. 77, no. 9, pp. 1189-1203, 2007.

[2] Y. Zhu and K. Tomsovic, "Optimal Distribution Power Flow for Systems with Distributed Energy Resources," Int. J. Elect. Power Energy Syst., vol. 29, no. 3, pp. 260-267, Mar. 2007.

[3] S. Gill, I. Kockar and G.W. Ault, "Dynamic Optimal Power Flow for Active Distribution Networks," IEEE Trans. Power Syst., vol. 29, no. 1, pp. 121-131, Jan. 2014.

[4] N. Daratha, B. Das and J. Sharma, "Coordination Between OLTC and SVC for Voltage Regulation in Unbalanced Distribution System Distributed Generation," IEEE Trans. Power Syst. , vol. 29, no. 1, pp. 289-299, Jan. 2014.

[5] M. J. Dolan, E. M. Davidson, I. Kockar,G.W. Ault and S. D. J. McArthur, "Distribution Power Flow Management Utilizing an Online Optimal Power Flow Technique," IEEE Trans. Power Syst. vol. 27, no. 2, pp. 790-799, May 2012.

[6] S. Paudyal,C. A. Canizares and K. Bhattacharya, "Optimal Operation of Distribution Feeders in Smart Grids," IEEE Trans. Ind. Electron., vol. 58, no. 10, pp. 4495-4503, Oct. 2011.

[7] A. Borghetti, M. Bosetti, S. Grillo, S.Massucco, C. Nucci,M. Paolone, and F. Silvestro, "Short-term scheduling and control of active distribution systems with high penetration of renewable resources," IEEE Syst. J., vol. 4, no. 3, pp. 313-322, Sep. 2010.

[8] Q. Zhou and J.Bialek, "Generation curtailment to manage voltage constraints in distribution networks," IET Gener., Transm., Distrib., vol. 1, no. 3, pp. 492-498, May 2007.

[9] V. Calderaro, G. Conio, V. Galdi, G. Massa and A. Piccolo, "Optimal Decentralized Voltage Control for Distribution Systems With InverterBased Distributed Generators," IEEE Trans. Power Syst., vol. 29, no. 1, pp. 230-241, Jan. 2014.

[10] A. Borghetti, M. Bosetti, S. Grillo, M. Paolone, and F. Silvestro, "ShortTerm Scheduling of Active Distribution Systems," in Proc. 2009 IEEE Power Tech Conf., Bucharest, Romania, Jun.28-Jul. 32009.

[11] A. Keane and M. O'Malley, "Optimal Allocation of Embedded Generation on Distribution Networks," IEEE Trans. Power Syst., vol. 20, no. 3, pp. 1640-1646, Aug. 2005.

[12] Y. P. Agalgaonkar, B.C. Pal and R.A. Jabr, "Distribution Voltage Control Considering the Impact of PV Generation on Tap Changers and Autonomous Regulators," IEEE Trans. Power Syst., vol. 29, no. 1, pp. 182-192, Jan. 2014.

[13] D. Shirmohammadi, H. Hong, A. Semlyen, and G. Luo, "A compensation- based power flow method for weakly meshed distribution and transmission networks," IEEE Trans. Power Syst., vol. 3, no. 2, pp. 753-762, May 1988.

[14] M. Begovic and A. Phadke, "Control of voltage stability using sensitivity analysis," IEEE Trans. Power Syst., vol. 7, no. 1, pp. 114-123, Feb. 1992.

[15] Q. Zhou and J. Bialek, "Simplified calculation of voltage and loss sensitivity factors in distribution networks," in Proc. 16th Power Syst. Comput. Conf. (PSCC2008), Glasgow, U.K., 2008.

[16] K. Christakou, J. LeBoudec, M. Paolone and D. C. Tomozei, "Efficient computation of sensitivity coefficients of node voltages and line currents in unbalanced radial electrical distribution networks," IEEE Trans. Smart Grid, vol. 4, no. 2, pp. 741-750, Jun. 2013.

[17] G. Liu, Y. Xu, O. Ceylan and K. Tomsovic, "A new linearization method of unbalanced electrical distribution networks," in Proc. 46th North American Power Symposium (NAPS 2014), Pullman, WA, Sep. 2014.

[18] O. Ceylan, G. Liu, Y. Xu and K. Tomsovic, "Distribution system voltage regulation by distributed energy resources," in Proc. 46th North American Power Symposium (NAPS 2014), Pullman, WA, Sep. 2014.

[19] Distribution Test Feeders [online] Available: http://ewh.ieee.org/soc/pes/dsacom/testfeeders/. 